D.O.I.: $10.3895 / \mathrm{S} 1808-04482007000100011$

\title{
MÉTODO MULTICRITÉRIO DE APOIO À DECISÃO NA GESTÃO DA MANUTENÇÃO: APLICAÇÃO DO MÉTODO ELECTRE I NA SELEÇÃO DE EQUIPAMENTOS CRÍTICOS PARA PROCESSO
}

\section{MULTIPLE CRITERIA METHOD TO MAINTENANCE MANAGEMENT DECISION SUPPORT: APLICATION OF ELECTRE I METHOD TO PROCESS CRITIC EQUIPMENTS ELECTION}

\author{
Kurtt Schamne Helmann ${ }^{1}$; Rui Francisco Martins Marçal ${ }^{2}$ \\ ${ }^{1}$ Federal Technological University of Paraná - UTFPR - Ponta Grossa - Brasil \\ kurtt2006@pg.cefetpr.br \\ ${ }^{2}$ Federal Technological University of Paraná - UTFPR - Ponta Grossa - Brasil \\ marcal@utfpr.edu.br
}

\begin{abstract}
Resumo
O presente estudo trata da aplicação de métodos de apoio multicritério de apoio à decisão para auxiliar a Gestão da Manutenção. A determinação dos equipamentos críticos ao processo produtivo é um desafio dos gestores, uma vez que devem ser tratados de forma diferenciada a fim de evitar danos humanos, financeiros e ambientais à empresa, aos colaboradores e à sociedade. Através da aplicação do método ELECTRE I, baseando-se em critérios e pesos estabelecidos, são determinadas quais são os equipamentos críticos ao processo no cenário apresentado, auxiliando o gestor da manutenção na decisão.
\end{abstract}

Palavras-chave: gestão da manutenção; decisão multicritério; ELECTRE I.

\section{Introdução}

A manutenção desempenha um papel estratégico nas organizações competitivas, proporcionando a confiabilidade e a disponibilidade dos processos produtivos, evitando a deterioração e as falhas através da preservação dos equipamentos.

Para Kardec e Nascif (2005, p.23) a manutenção passou a desempenhar papel estratégico nas organizações modernas, sendo o diferencial das empresas líderes em seus segmentos. Segundo os autores, na visão atual, a manutenção existe para "garantir a disponibilidade dos equipamentos e instalações de modo a atender a um processo de produção ou de serviço, com confiabilidade, segurança, preservação do meio ambiente e custos adequados". 
Segundo Monchy (1989) a manutenção dos equipamentos de produção é um elemento chave tanto para a produtividade das industrias quanto para a qualidade dos produtos. Laugeni e Martins (2002) corroboram com essa idéia colocando que as interrupções levam quase sempre a uma queda na qualidade. Máquinas com defeito trabalhando de forma inadequada, não fabricam produtos dentro das especificações previstas. Este movimento mundial em busca de maior qualidade e menor custo tem levado as empresas a dar à manutenção uma atenção toda especial.

As paradas não programadas da linha de produção geram a queda na receita, se convertendo em prejuízos significativos. Com a ocorrência de paradas, eleva-se o custo da produção, diminuindo a produtividade, o lucro e comprometendo a qualidade dos produtos em processo.

A competitividade de uma empresa está diretamente ligada à qualidade dos serviços prestados pelas equipes de manutenção, que por sua vez depende da disponibilidade. Os problemas variam muito dependendo do tipo de atividade, os quais são enumerados a seguir (SOUZA, 2002).

Torna-se fundamental para o sucesso da manutenção, cumprindo com seu papel junto ao processo produtivo, o planejamento e controle da manutenção (PCM), onde de forma planejada e organizada a equipe passa a intervir nos equipamentos. Dentro do PCM uma das etapas essenciais é a determinação dos equipamentos críticos ao processo, os quais necessitam de atenção especial, seja no projeto, na instalação ou na determinação das manutenções. O papel do Gestor da Manutenção é a classificação desses equipamentos, o que muitas vezes não é uma tarefa fácil, uma vez que envolve múltiplos critérios. O processo decisório da manutenção, desenvolvido nas empresas, tem se caracterizado por uma abordagem informal sem embasamento quantitativo e metodológico. Embora existam várias contribuições disponíveis na literatura, no sentido de utilizar-se de modelos quantitativos, ainda há problemas para os quais um método de abordagem não foi proposto na manutenção (CAVALCANTE e COSTA, 2003).

Diante do exposto, o presente estudo propõe como forma de auxílio ao Gestor de Manutenção no momento da decisão para a seleção de equipamentos críticos no processo produtivo, a utilização da metodologia de apoio à decisão ELECTRE I.

\section{Processo decisório}

A tomada de decisão é um fato do dia a dia, presente em todas as atividades desenvolvidas pelo homem. Naturalmente as pessoas enfrentam situações que lhes exigem algum tipo de decisão. Nestas situações apresentam-se vários caminhos ou alternativas de ações possíveis e dentre estas se deve optar por aquela que melhor satisfaz os objetivos em causa.

Alguns autores afirmam que decidir é posicionar-se em relação ao futuro. Gomes et al (2002, p. 12) definem decisão como "o processo de colher informações, atribuir importância a elas, 
posteriormente buscar possíveis alternativas de solução e, depois, fazer a escolha entre alternativas".

Segundo Zeleny apud Gomes et al (2002, p.12), “a tomada de decisão é um esforço para tentar resolver problemas de objetivos conflitantes, cuja presença impede a existência da solução ótima e conduz à procura do melhor compromisso".

O processo de tomada de decisão pode ser percebido nas mais simples tarefas enfrentadas pelo ser humano. A compra de bens de consumo, até a escolha de uma instituição de ensino, são problemas enfrentados diariamente pelas pessoas. A solução para muitos desses tipos de problemas baseia-se exclusivamente em um único parâmetro de decisão - por exemplo, o custo.

Entretanto, muitos problemas complexos são submetidos à decisão das pessoas. Os problemas complexos, foco do presente estudo, caracterizam-se por apresentar ao menos dois parâmetros, em geral conflitantes, que afetam a decisão. De acordo com Gomes et al (2002) a tomada de decisões complexas é uma das mais difíceis tarefas enfrentadas individualmente ou por grupos, pois invariavelmente, tais decisões devem atender múltiplos objetivos e seus impactos não podem ser claramente identificados.

Souza (2002) afirma que uma boa decisão é uma conseqüência lógica daquilo que se quer, daquilo que se sabe e daquilo que se pode fazer, a saber, que:

- O que se quer se refere às preferências que se têm pelas diversas conseqüências das decisões. Tais conseqüências podem ser incertas ou distribuídas ao longo do tempo.

- O que se sabe é o conhecimento em relação às grandezas envolvidas no processo e das relações entre elas; a informação advinda do processo de decisão; a percepção das circunstâncias e das leis que a afetam.

- O que se pode fazer são as alternativas disponíveis e possíveis de ação.

O processo decisório não é único, possuindo suas especificidades para cada caso. Nas diversas áreas, o gestor deve estar ciente das etapas de um processo decisório, assim como dos passos para uma decisão eficaz com o objetivo de buscar subsídios para uma decisão inteligente, pautada em critérios consistentes.

\section{Apoio à decisão multicritério}

Os problemas complexos de tomada de decisão são comuns nas diversas áreas. Assim como em outras áreas, na manutenção desde tempos mais remotos o homem tenta resolvê-los, apoiandose em abstrações, heurísticas e raciocínios dedutivos, com o objetivo de orientar e validar as suas escolhas (GOMES, ARAYA e CARIGNANO, 2004). 
A decisão apresenta-se como uma das mais importantes atividades dos gestores em todas as áreas de atuação. $\mathrm{Na}$ manutenção industrial, a todo o momento os gestores deparam-se com situações que exigem decisões, as quais são carregadas de complexidade. De acordo com Marçal e Susin (2005), mantenedores, engenheiros, enfim profissionais de manutenção, deparam-se atualmente com as mais complexas tarefas na atividade de manutenção e para se assegurarem de cumprir tais tarefas assertivamente, os profissionais buscam uma série de ferramentas que os auxiliem no processo de tomada de decisões.

De modo geral, problemas complexos de tomada de decisão, de acordo com Gomes, Araya e Carignano (2004, p. 1) apresentam pelo menos uma das características a seguir:

- Os critérios para resolução do problema são conflitantes entre si.

- Tanto os critérios como as alternativas não estão claramente definidas, e as conseqüências da escolha de uma alternativa em relação a pelo menos um critério, não são devidamente compreendidas.

- Os critérios e as alternativas podem estar interligados, de forma que um critério pode refletir nos demais.

- A solução dos problemas depende de um conjunto de pessoas, cada uma com pontos de vistas próprios, em geral conflitantes.

- As restrições dos problemas não estão bem definidas, podendo existir dúvidas em relação ao que é critério e ao que é restrição.

- Existem critérios quantificáveis, e outros somente o são por meio de juízos de valor efetuados sobre uma escala.

- A escala para um critério pode ser cardinal, verbal ou ordinal, dependendo da disponibilidade de dados e da natureza dos critérios.

Até a primeira metade do século XX, utilizava-se apenas a esperança matemática para a tomada de decisões em condições aleatórias. Porém em muitas situações, observa-se que o risco associado a tal procedimento não era aceitável. Somente a partir do final da Segunda Guerra Mundial, com a experiência adquirida pelas tropas aliadas em relação à solução de problemas logísticos militares, é que um grande número de instituições de pesquisa dedicaram-se à análise e à preparação de decisões, utilizando-se da Pesquisa Operacional. (GOMES, ARAYA e CARIGNANO, 2004).

De acordo com Chiavenato (1999, p. 716) a Pesquisa Operacional é "a aplicação de métodos, técnicas e instrumentos científicos a problemas que envolvem as operações de um sistema, de modo a proporcionar, aos que controlam o sistema, soluções ótimas para o problema”. 
A Pesquisa Operacional faz parte de um processo de aperfeiçoamento matemático para solução de problemas de decisões complexos, onde o agente da decisão faz uso de múltiplos critérios para auxiliá-lo no processo decisório.

As técnicas de análise multicritério tiveram seu surgimento nas décadas de 70 e 80 , em substituição aos modelos ortodoxos de pesquisa operacional, que surgiram na década de 50 para a resolução de problemas logísticos-militares nas forças armadas durante a $2^{\mathrm{a}}$ Guerra Mundial, que buscavam soluções para problemas gerenciais complexos (MARINS e COZENDEY, 2005).

Desde o seu surgimento, o estudo de problemas de decisão que estão inseridos em um ambiente complexo tem sido objeto de preocupação de pesquisadores sobre o assunto. São destacados a existência de alguns métodos aplicados aos problemas de decisão com múltiplos critérios, dentro da área de Pesquisa Operacional, de onde surgiu o campo de estudo: Apoio Multicritério a Decisão.

A abordagem multicritério de apoio à decisão pode ser caracterizada como um conjunto de métodos que buscam tornar claro um problema, no qual as alternativas são avaliadas por múltiplos critérios, os quais na maioria dos casos são conflitantes (GOMES et al, 2002). De acordo com Diehl apud Marins e Cozendey (2005), esse tipo de abordagem não apresenta uma solução ideal para os problemas, mas entre todas as possíveis, a mais coerente com a escala de valores e o método utilizado.

Os métodos de apoio multicritério à decisão têm um lado científico, mas ao mesmo tempo, subjetivo, apresentando consigo a capacidade de agregar todas as características consideradas importantes, inclusive as não quantitativas, com o objetivo de permitir a transparência e a sistematização do processo referente aos problemas de tomada de decisões (GOMES, ARAYA e CARIGNANO, 2004).

Esses métodos multicritérios fazem um enfoque diferenciado sobre os problemas e passam a atuar sobre a forma de auxílio à decisão, apresentando segundo Gomes, Araya e Carignano (2004, p.3) algumas características bem definidas em relação à sua metodologia:

- A análise do processo de decisão, em que essa metodologia é aplicada, tem sempre o objetivo de identificar informações/regiões críticas.

- A existência de uma melhor compreensão acerca das dimensões do problema.

- A possibilidade de haver diferentes formulações válidas para um único problema.

- A aceitação de que, em problemas complexos, as situações nem sempre se ajustam a um perfeito formalismo e, em particular, de que estruturas que representam de forma parcial a compatibilidade entre as alternativas podem ser relevantes no processo de auxílio à decisão. 
- O uso de representações explícitas de uma estrutura de preferências, em vez de representações numéricas definidas artificialmente, muitas vezes pode ser mais apropriado a um problema específico de tomada de decisões.

Almeida e Costa (2003) colocam que o apoio multicritério tem como princípio, no processo de decisão, buscar o estabelecimento de uma relação de preferências entre as alternativas que estão sendo avaliadas sob a influência de vários critérios. Complementa Gomes, Araya e Carignano (2004) que o estudo de problemas de decisões, a partir do enfoque multicritério, não objetiva apresentar ao decisor uma solução específica para o problema, mas sim, apoiar o processo de decisão ao recomendar ações ou cursos de ação a quem vai tomar a decisão.

Existem vários métodos desenvolvidos para a abordagem e tratamento de problemas com múltiplos critérios. Destacam-se dois grupos representativos de escolas citados na literatura (ALMEIDA e COSTA, 2003; GOMES, ARAYA e CARIGNANO, 2004):

- Escola americana: destaca-se a teoria multiatributo (MAUT).

- Escola européia: destacam-se os métodos de sobreclassificação, em especial os da família ELECTRE e família PROMETHEE.

Outras abordagens ou métodos são apresentados na literatura: programação matemática multiobjetivo, SMART, AHP, MACHBETH, TODIM (ALMEIDA E COSTA, 2003).

A escolha do método vai depender de vários fatores destacando-se as características: do problema analisado, do contexto considerado, da estrutura de preferências do decisor e da problemática em si (ALMEIDA e COSTA, 2003).

As soluções dos problemas de decisão variam em função do resultado pretendido. Determinado um problema, segundo Gomes, Araya e Carignano (2004) uma das seguintes problemáticas é abordada, conforme exposto na tabela 1:

Tabela 01 - Problemática em função do tipo de problema.

\begin{tabular}{|c|c|}
\hline Tipo do Problema & Problemática abordada \\
\hline Tipo $\alpha(\mathrm{P} \alpha)$ & $\begin{array}{l}\text { Selecionar a "melhor" alternativa ou as melhores } \\
\text { alternativas. }\end{array}$ \\
\hline Tipo $\beta(\mathrm{P} \beta)$ & $\begin{array}{l}\text { Aceitar alternativas que parecem "boas" e descartar as } \\
\text { que parecem "ruins", ou seja, realizar uma classificação } \\
\text { das alternativas. }\end{array}$ \\
\hline Tipo $\gamma(\mathrm{P} \gamma)$ & Gerar uma ordenação das alternativas. \\
\hline Tipo $\sigma(P \sigma)$ & Realizar uma descrição das alternativas. \\
\hline
\end{tabular}

Aspectos ligados às preferências do decisor são geralmente considerados para a escolha de um método de apoio à decisão. Em determinadas circunstâncias, simplicidade e facilidade de operacionalização podem ser fatores cruciais para solucionar o problema. Muitas vezes, a falta de 
intimidade com outras metodologias implica ao decisor a escolha de um determinado método, o qual nem sempre é o mais apropriado para a situação.

De acordo com Almeida e Costa (2003), a aplicação de qualquer método de análise multicritério pressupõe a necessidade de se estabelecer quais objetivos o decisor pretende alcançar, estabelecendo a representação destes múltiplos objetivos através do uso de múltiplos critérios.

\section{Aplicação do método multicritério ELECTRE I para seleção de equipamentos críticos para o processo}

Tratando o problema de seleção de equipamentos críticos para o processo, como uma problemática do tipo $\mathrm{P} \alpha$, ou seja, de escolha da melhor ou melhores alternativas, será utilizado o método de sobreclassificação ELECTRE I.

Segundo Gomes, Araya e Carignano (2004), o método ELECTRE I procura esclarecer a decisão por intermédio da escolha de um subconjunto que seja restrito o máximo possível e que contenha as melhores alternativas.

O método ELECTRE I constrói as relações de sobreclassificação a partir de índices de concordância e discordância, mediante comparações entre pares de equipamentos (ALMEIDA e COSTA, 2003).

Dentro do processo produtivo existe uma infinidade de equipamentos que podem afetá-lo de forma mais ou menos significativa. Para exemplificar o modelo proposto determina-se a seleção entre seis equipamentos quaisquer de um processo de fabricação de embalagens de polpa moldada, sendo identificados como equipamento 01, 02 e assim sucessivamente até equipamento 06.

A decisão do gestor deverá ocorrer entre as alternativas expostas, sendo os seguintes critérios analisados para a tomada de decisão: riscos de segurança ao ser humano e às instalações, riscos ao meio-ambiente e perdas de produção. A tabela 2 propõe uma avaliação suposta para cada alternativa em relação a cada critério, além da determinação de pesos para os critério.

Tabela 02 - Desempenho das Alternativas nos Critérios e Pesos dos Critérios.

\begin{tabular}{cccc}
\hline Alternativas & \multicolumn{2}{c}{ Critérios } & \\
\cline { 2 - 4 } & Perdas de Produção & Riscos à Segurança & Riscos ao meio-ambiente \\
\hline $\begin{array}{c}\text { Equipamento 01 - Bomba } \\
\text { de Vácuo }\end{array}$ & 0,7 & 0,8 & 0,2 \\
$\begin{array}{c}\text { Equipamento 02 - Bomba } \\
\text { de Água Fresca }\end{array}$ & 0,8 & 1,0 & 0,5 \\
$\begin{array}{c}\text { Equipamento 03 - Bomba } \\
\text { de Água Recuperada }\end{array}$ & 0,5 & 1,0 & 0,8 \\
Equipamento 04 - Redutor & 0,7 & 0,9 & 0,6
\end{tabular}




\begin{tabular}{|c|c|c|c|}
\hline $\begin{array}{c}\text { Equipamento } 05-\text { Bomba } \\
\text { de Massa }\end{array}$ & 0,1 & 0,8 & 0,7 \\
\hline $\begin{array}{l}\text { Equipamento } 06- \\
\text { Ventilador de Secagem }\end{array}$ & 0,3 & 0,6 & 1,0 \\
\hline Pesos & 2 & 5 & 3 \\
\hline
\end{tabular}

Com os valores e pesos de cada critério para cada alternativa esses valores devem ser normalizados. Toma-se como método de normalização o quociente entre o elemento a ser normalizado e a soma total, obtendo-se a tabela 3.

Tabela 03 - Desempenhos Normalizados das Alternativas nos Critérios e Pesos Normalizados dos Critérios.

\begin{tabular}{cccc}
\hline Alternativas & \multicolumn{3}{c}{ Critérios } \\
\cline { 2 - 4 } & Perdas de Produção & Riscos à Segurança & Riscos ao meio-ambiente \\
\hline Equipamento 01 & 0,226 & 0,157 & 0,053 \\
Equipamento 02 & 0,258 & 0,196 & 0,132 \\
Equipamento 03 & 0,161 & 0,196 & 0,211 \\
Equipamento 04 & 0,226 & 0,176 & 0,158 \\
Equipamento 05 & 0,032 & 0,157 & 0,184 \\
Equipamento 06 & 0,097 & 0,118 & 0,263 \\
\hline Pesos & 0,2 & 0,5 & 0,3 \\
\hline
\end{tabular}

Após a normalização dos critérios e pesos são calculados os índices de concordância com a equação 1:

$$
\begin{array}{|c|}
\mathrm{c}_{\mathrm{i}, \mathrm{k}}=\sum_{\mathrm{wj}} \\
\mathrm{j \in c}(\mathrm{xi}, \mathrm{xk})
\end{array}
$$

Equação 1

Onde wj $>0$ representa o peso normalizado do critério j (dividindo pela soma total dos pesos), de maneira que $\mathrm{n}$ $\Sigma_{w j}=1 \cdot$

$j=1$

É importante salientar que os três critérios devem ser minimizados. Realizando-se os cálculos para todos os pares de alternativas possíveis obtém-se a matriz $\mathrm{C}$, que agrupa todos os índices de concordância. A figura 01 apresenta a matriz C. 
Figura 01 - Índices de Concordância em relação às alternativas.

\begin{tabular}{cccccccc|}
\hline & $C$ & 1,0 & & 0,8 & 1,0 & 0,8 & 0,3 \\
0 & & & 0,8 & 0,3 & 0,3 & 0,3 \\
0,2 & & 0,7 & & 0,2 & 0 & 0,3 \\
0,2 & 0,7 & 0,8 & & 0 & 0,3 \\
0,7 & 0,7 & 1,0 & 0,7 & & 0,5 \\
0,7 & 0,7 & 0,7 & 0,7 & 0,5 & \\
\hline
\end{tabular}

Em seguida são calculados também os índices de discordância, utilizando a equação 2:

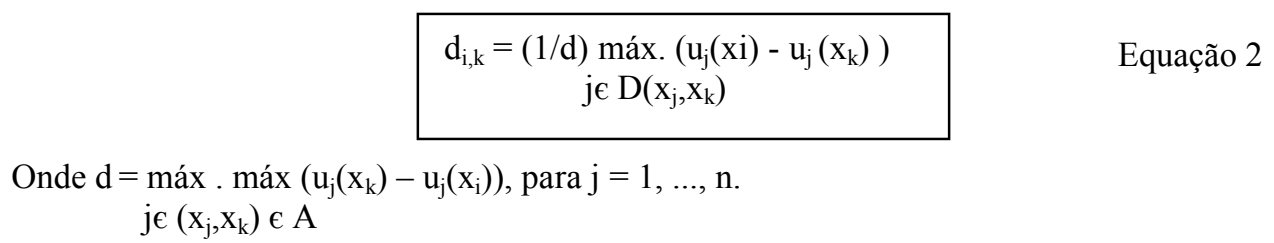

Efetuando os cálculos para todos os pares de alternativas, obtém-se a matriz D expressa na figura 02, que contém os índices de discordância para todas as alternativas.

Figura 02 - Índices de Discordância em relação às alternativas.

$\mathrm{D}=\quad$\begin{tabular}{ccccccc|}
\hline & 0 & 0,288 & 0 & 0,858 & 0,571 \\
1 & & 0,429 & 0,142 & 1 & 0,712 \\
0,699 & 0,350 & & 0,235 & 1 & 0,345 \\
0,465 & 0,115 & 0,288 & & 0,858 & 0,571 \\
0,580 & 0,230 & 0 & 0,115 & & 0,173 \\
0,929 & 0,580 & 0,230 & 0,465 & 0,350 & \\
\hline
\end{tabular}

Uma vez obtida as matrizes de concordância e discordância o tomador de decisão estabelece os limiares de concordância e discordância de acordo com as suas preferências. Isso significa que a melhor ou melhores alternativas devem atender que $\mathrm{c}_{\mathrm{i}, \mathrm{k}}>\mathbf{c}$ e $\mathrm{d}_{\mathrm{i}, \mathrm{k}}<\mathbf{d}$. É possível ao decisor, através da variação dos limiares de concordância e discordância, obter a alternativa, ou conjunto de alternativas que mais atende as preferências do decisor, conforme mostra a tabela 4 . 
Tabela 04 - Resultado das melhores alternativas para diferentes valores dos limites de concordância e discordância.

\begin{tabular}{ccc}
\hline Concordância (c) & Discordância (d) & Melhores alternativas \\
\hline 0,3 & 0,7 & 5 \\
0,4 & 0,6 & 5 \\
0,5 & 0,5 & - \\
0,6 & 0,4 & - \\
\hline
\end{tabular}

Como já dito anteriormente, o método ELECTRE I procura auxiliar a resolver problemas de seleção. Partindo dos limiares estabelecidos, a melhor alternativa de decisão para o cenário estudado é a alternativa 5, ou seja, considerando o contexto proposto o equipamento mais crítico ao processo, em relação aos critérios de perdas de produção, riscos à segurança e riscos ao meioambiente é a bomba de massa.

\section{Conclusões}

Este estudo teve como objetivo a aplicação de métodos de apoio à decisão multicritério para a seleção de equipamentos críticos ao processo produtivo, à luz dos critérios estabelecidos pelo decisor, dentro do gerenciamento da manutenção.

Os resultados obtidos com a aplicação da metodologia ELECTRE I ao processo decisório no cenário específico demonstram a utilidade dos métodos multicritérios de apoio à decisão. É importante destacar que a aplicação neste trabalho não pode ser generalizada, sendo necessárias adaptações para cada situação específica.

Tem-se como sugestão a ampliação do estudo através da utilização de outros critérios, como por exemplo, a disponibilidade de mão-de-obra e a política da empresa, entre outros, assim como a ampliação da abrangência do número de equipamentos do processo. Além disso, observa-se que com a aplicação do método ELECTRE I não fica estabelecido ordenação entre as alternativas, ou seja, não fica estabelecido qual equipamento é o mais crítico no processo. Desta forma se faz necessário a utilização de outros métodos que tratem a problemática como uma problemática de ordenação, como é o caso do método ELECTRE II.

\footnotetext{
Abstract

The present study treats application to multiple criteria method to maintenance management decision support. The determination of the critical equipment to the productive process is a challenge of the managers, a time that must be dealt with differentiated form in order to prevent human, financial and ambient damages to the company, to the collaborators and the society. Through the application of method ELECTRE I, being based on criteria and weights established,
} 
we determined which the critical equipment to the process are in the presented scene, supporting manager decision.

Key-words: maintenance management; multiple criteria decision; ELECTRE I.

\section{Referências}

ALMEIDA, Adiel T. de. \& COSTA, Ana Paula C. S. Aplicações com métodos multicritério de apoio à decisão. Recife: Universitária da UFPE, 2003.

CAVAlCANTE, C. A. V. \& ALMEIDA, A. T de. Modelo multicritério de apoio a decisão para o planejamento de manutenção preventiva utilizando PROMETHEE II em situações de incerteza. Pesquisa Operacional. Vol. 25, número 2, p.279-296, mai-ago, 2005.

CHIAVENATO, Idalberto. Introdução à teoria geral da administração. $5^{\text {a }}$ Edição, Rio de Janeiro: Campus, 1999.

GOMES, L. F. M. A.; ARAYA, M. C. G. \& CARIGNANO, C. Tomada de decisões em cenários complexos. São Paulo: Pioneira, 2004.

GOMES, L. F. M. A.; GOMES, C. F. S. \& ALMEIDA, A. T. Tomada de decisão Gerencial: Enfoque Multicritério. Rio de Janeiro: Atlas, 2002.

KARDEC, Alan \& NASCIF, Júlio. Manutenção função estratégica. Rio de Janeiro, Qualitymark, 2005.

MARÇAL, Rui F. M., SUSIN, Altamiro A. Predizendo Falhas em Sistemas Rotativos usando para o diagnóstico lógica Fuzzy. Anais $20^{\circ}$ Congresso Brasileiro de Manutenção - ABRAMAN. Belo Horizonte, 2005.

MARINS, Cristiano S. \& COZENDEY, Manaara I. A metodologia de multicritério como ferramenta para tomada de decisões gerenciais: um estudo de caso. In: $25^{\circ}$ Encontro Nacional de Engenharia de Produção (ENEGEP). Anais. Porto Alegre / RS, 2005.

MONCHY, François. A Função Manutenção - Formação para a Gerência da Manutenção Industrial. São Paulo: Editora Durban, 1989.

SOUZA, Fernando. M. C. de. Decisões racionais em situações de incerteza. Recife: Universitária da UFPE, 2002.

\section{Dados dos autores:}

Nome completo: Kurtt Schamne Helmann

Filiação institucional: Universidade Tecnológica Federal do Paraná (UTFPR)

Departamento: Programa de Pós-graduação em Engenharia de Produção (PPGEP)

Função ou cargo ocupado: Mestrando em Engenharia de Produção

Endereço completo para correspondência: Avenida dos Pioneiros, 2868, centro Carambei - PR

Telefones para contato: (42) 32318500

e-mail: kurtt_2006@pg.cefetpr.br

Nome completo: Rui Francisco Martins Marçal

Filiação institucional: Universidade Tecnológica Federal do Paraná (UTFPR)

Departamento: Programa de Pós-graduação em Engenharia de Produção 
Função ou cargo ocupado: Professor

Endereço completo para correspondência: Avenida Monteiro Lobato, Jardim Pitangui, 84016-210

Telefones para contato: (42) 32204805

e-mail: marcal@utfpr.edu.br

Recebido para publicação em: 18/10/2006

Aceito para publicação em: 27/02/2007 